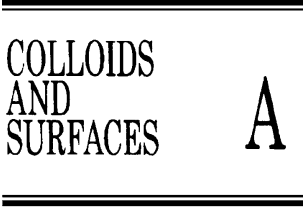

Colloids and Surfaces A: Physicochem. Eng. Aspects 213 (2003) 167-173

www.elsevier.com/locate/colsurfa

\title{
Caging effects in amorphous hard-sphere solids
}

\author{
Albert P. Philipse* \\ Van't Hoff Laboratory for Physical and Colloid Chemistry, Debye Institute, Utrecht University, Padualaan 8, 3584 CH Utrecht, The \\ Netherlands
}

\begin{abstract}
The caging density of a $d$-dimensional hard-sphere solid is introduced as the density at which the average contact number on a sphere equals the caging number, defined as the average minimal number of randomly parked neighbors which completely arrest (cage) a test sphere. The mean-field caging density, which is consistent with random (loose) sphere packing in $d=2$ and $d=3$, parallels the (always higher) $d$-dimensional densities of regular close packed crystals. The gap between caging and close-packing densities is ascribed to a difference in geometric optimization. This difference also explains the gap between sphere co-ordination in packings or glasses of colloidal spheres and the absolute maximum for close-packed crystals. Caging numbers appear to be an important feature of random packings in general since caging effects also account for the particle shape dependence of random thin-rod packings.

(C) 2002 Elsevier Science B.V. All rights reserved.
\end{abstract}

Keywords: Caging density; Hard-sphere solid; Thin-rod packings; Colloidal hard spheres; Colloidal glasses

\section{Introduction}

Studying the geometry of non-crystalline, amorphous sphere solids is of interest to better understand random packings or glasses of hard-sphere colloids. Such systems occur in a kinetic glass transition, at which structural rearrangements in colloidal hard-sphere fluids [1-10] freeze rapid enough to bypass nucleation of a crystal phase. Instead an arrested solid-like glass state is formed which preserves much of the fluid structure as this structure resembles that of a random sphere packing. This continuity of the transition from hard-sphere fluids to amorphous solids suggests an

* Tel.: +31-30-253-2391; fax: +31-30-253-3870.

E-mail address: fccoffice@chem.uu.nl (A.P. Philipse). alternative to the usual liquid-state perspective [25] on the sphere-glass (a perspective which inevitably involves complicated dynamics), namely a solid-state viewpoint comprising a purely geometrical account of arrested ('caged') spheres. Such an account is further motivated by the insight that the progressive structural arrest of hard spheres on approach of a glass transition is ultimately caused by purely geometrical restrictions $[9,10]$. Usually these restrictions are qualitatively described as slowly fluctuating neighbor cages which trap spheres over increasing time intervals at increasing densities [1]. In what follows we disregard any thermal fluctuations and consider a static snapshot of hard spheres, or an amorphous sphere solid at zero temperature. The idea is to find the solid density from a geometric optimization problem 
based on a precise definition of a static sphere cage.

This optimization problem is suggested by the radical difference between the geometry of amorphous and regular close-packed sphere solids. In the latter case each sphere is touched by the largest possible number (the 'kissing number') of twelve contacting neighbors [11]. Moreover, the density $\pi / \sqrt{18}=0.7405 \ldots$ of the close-packed crystal is the highest possible global density for a threedimensional sphere packing; Keppler's famous conjecture proven recently by Hales [11]. What sort of optimization could an amorphous sphere solid (or static glass) represent? A global density maximization, now under the constraint that the structure remains random, is unlikely when caging effects dominate; in a rapid density quench spheres simply lack time and mobility to increase contact numbers much above what is minimally needed to form a sphere cage (Figs. 1 and 2). Thus we consider an amorphous sphere solid density which, in contrast to the global maximization of the hardsphere crystal, derives from local minimization. The relevant problem is to find the average volume fraction (denoted here as the caging density) implied by the complete arrest or caging of spheres by the on average minimal number of contacts with random neighbors.

\section{The caging number}

First we will briefly recapitulate the concept of a caging number introduced and discussed in detail elsewhere [16]. Caging is defined here as the situation in which a test sphere $T$ has no translational degrees of freedom. A cage is constructed by placing fixed contact points at arbitrary positions on the surface of $T$. A minimal cage is formed for $N$ contacts that cage $T$, whereas $N-1$ contacts do not cage the test sphere. By definition $T$ is uncaged whenever an equator can be chosen on $T$ such that all point contacts are located on the same hemisphere of $T$. The caging number is defined as the expectation value $\left\langle N_{d}\right\rangle$, obtained from a large number of repeated caging experiment on a sphere in Euclidean dimension $d$. An analytical solution for $\left\langle N_{d}\right\rangle$ has been derived for contacts, which are completely uncorrelated due to the fact that the spheres which contact $T$ may overlap. The result for arbitrary dimension $d \geq 1$ is [16]:

$\left\langle N_{d}\right\rangle=2 d+1$

When the neighbours of $T$ are hard spheres the contact distribution on $T$ is subject to the constraint that no two spheres should overlap. There is a trivial solution in this case for one-dimensional spheres (Fig. 2(A)), namely $\left\langle N_{1}\right\rangle=2$, but no caging numbers have been calculated yet for higher dimension. However, computer simulation results of caging experiment on a $d$-dimensional hard sphere can be fitted accurately with [16]:

$\left\langle N_{d}\right\rangle=0.046 d^{2}+1.22 d+0.73$

In these simulations [16] the same construction of a sphere cage was used as in Fig. 1, namely random parking of hard spheres and discarding any parking which produces overlap. This constraint random packing is the only randomness that enters into the caging number. Note that the caging number for hard spheres in Eq. (2) falls below the value from Eq. (1). This is because the non-overlap constraint pushes contacts apart on the surface of $T$, so less contacts are needed to cage $T$ than in the case of uncorrelated contacts. The caging number is a well-defined expectation value, which - within a mean field approximation - can be converted to a well-defined density as follows.

\section{Caging density}

Consider a $d$-dimensional test sphere $T$ with diameter $\sigma$ and equal sized neighbor spheres $S$ with their center at a $d$-dimensional position vector $\boldsymbol{r}_{d}$ relative to the center of $T$. The function $f\left(r_{d}\right)$ equals unity when $T$ and $S$ have a region of overlap and is zero otherwise: $f\left(r_{d}\right)=0$ if $r_{d}>\sigma$, and $f\left(r_{d}\right)=1$ if $0 \leq r_{d} \leq \sigma$. The number of overlaps on $T$ by neighbors $S$ is therefore the $d$-fold integral:

$C_{d}=\int f\left(\boldsymbol{r}_{d}\right) \rho\left(\boldsymbol{r}_{d}\right) \mathrm{d} \boldsymbol{r}_{d}$ 


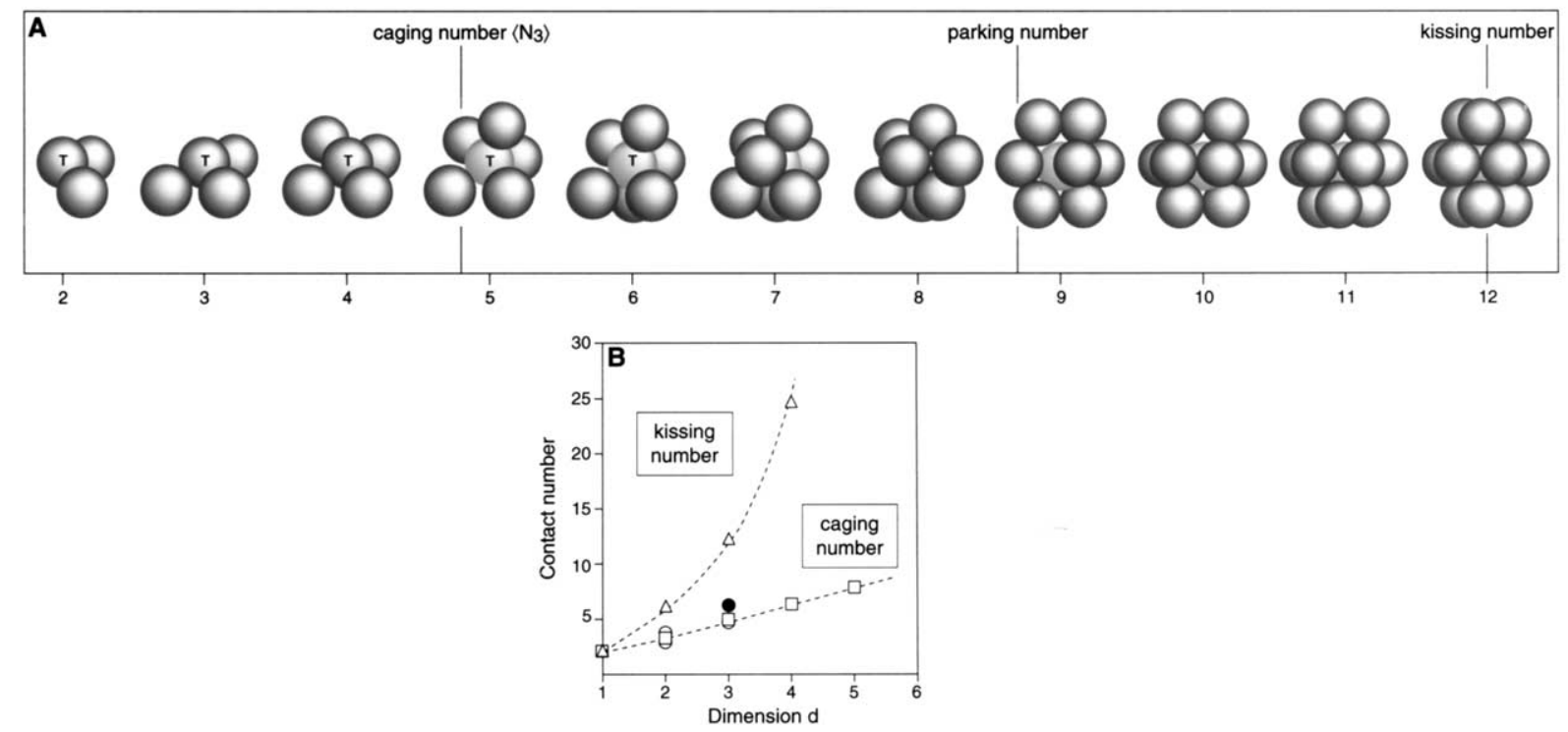

Fig. 1. (A) Co-ordination of a 3- $d$ test sphere $T$ starts with random parking of neighbors at fixed contact positions on $T$ under the constraint that no two spheres interpenetrate. When all contacts share the same hemisphere of $T$, the test sphere is uncaged, i.e. free to translate in some direction. Cages are formed by the minimal number of neighbors which completely arrests $T$ (which turns red). The average of this minimum is the caging number [16] $\left\langle N_{3}\right\rangle=4.79$. Random parking is continued until $T$ accommodates the maximal number of random, fixed contacts, the average of which equals the parking number [19]. The contact number $C$ can only be further increased by rearranging neighbors to ordered positions, with the single-valued kissing number $C=12$ of the FCC-crystal as the absolute maximum [11,21]. (B) The gap between ( $\square$ ) caging number from Eq. (2) and ( $\Delta$ ) kissing number [21] rapidly increases with dimension $d$, despite the modest density gap between sphere-glasses and densest lattice packing (Fig. 3). Apparently a sphere is only caged by a small fraction of a large number of spheres in its vicinity from which contacts are recruited to crystallize the amorphous solid. Shown are also average contact numbers for $3-d$ random close sphere packings from experiments $[16,17](\bigcirc)$ and simulations [18] (O), and for random 2- $d$ disc packings[14] ( $\bigcirc)$. These results show that contacts in random packings are close to caging numbers.

where $\rho\left(\boldsymbol{r}_{d}\right)$ is the local number density distribution of neighbors near $T$. The mean-field approximation is that the average of $C_{d}$ over all spheres only depends on the overall average number density $\bar{\rho}_{d}$ of the sphere system:

$$
\left\langle C_{d}\right\rangle=\bar{\rho}_{d} \int f\left(\boldsymbol{r}_{d}\right) \mathrm{d} \boldsymbol{r}_{d}
$$

The integral in Eq. (4) is by definition the excluded volume $V_{\mathrm{ex}, d}$, i.e. the region in which the center of $S$ must be located in order to overlap $T$. Thus the mean-field number density is simply the average number of overlaps counted in the excluded volume. For the infinitely hard spheres in this study, such overlaps shrink to zero-dimensional contact points on the surface of $T$. For this case we refer to $\left\langle C_{d}\right\rangle$ as the average contact number, rather than an average overlap number. The volume $V_{d}$ of a hyper sphere with diameter $\sigma$ is [21]:

$V_{d}=\frac{\pi^{d / 2}}{(d / 2) \Gamma(d / 2)}(\sigma / 2)^{d}$

where $\Gamma(x)$ is the gamma-function of $x$. It follows from the definition of $f\left(r_{d}\right)$ that $V_{\mathrm{ex}, d}$ is a hyper sphere with diameter $2 \sigma$. Therefore, $V_{\mathrm{ex}, d} / V_{d}=$ $2^{-d}$ and Eq. (4) leads to:

$\Phi_{d}=\left\langle C_{d}\right\rangle 2^{-d}$

Here $\Phi_{d}=\bar{\rho}_{d} V_{d}$ is the fraction of space occupied by the hyperspheres: $\Phi_{2}$ is a surface fraction of discs on a two-dimensional plane, $\Phi_{3}$ is the three-dimensional sphere volume fraction, etc. We define an amorphous solid (see also Fig. 2) to be at its caging density when the average contact number precisely equals the caging number $\left\langle N_{d}\right\rangle$ :

$\Phi_{d}=\left\langle N_{d}\right\rangle 2^{-d}$ 
(A)

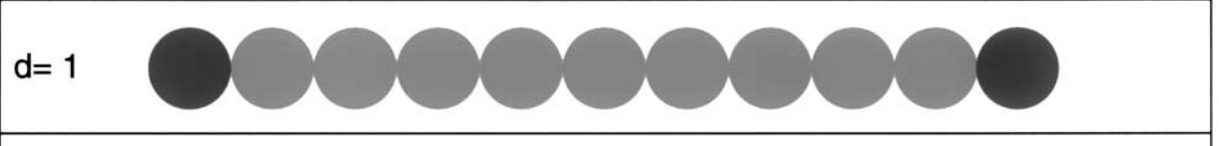

(B)

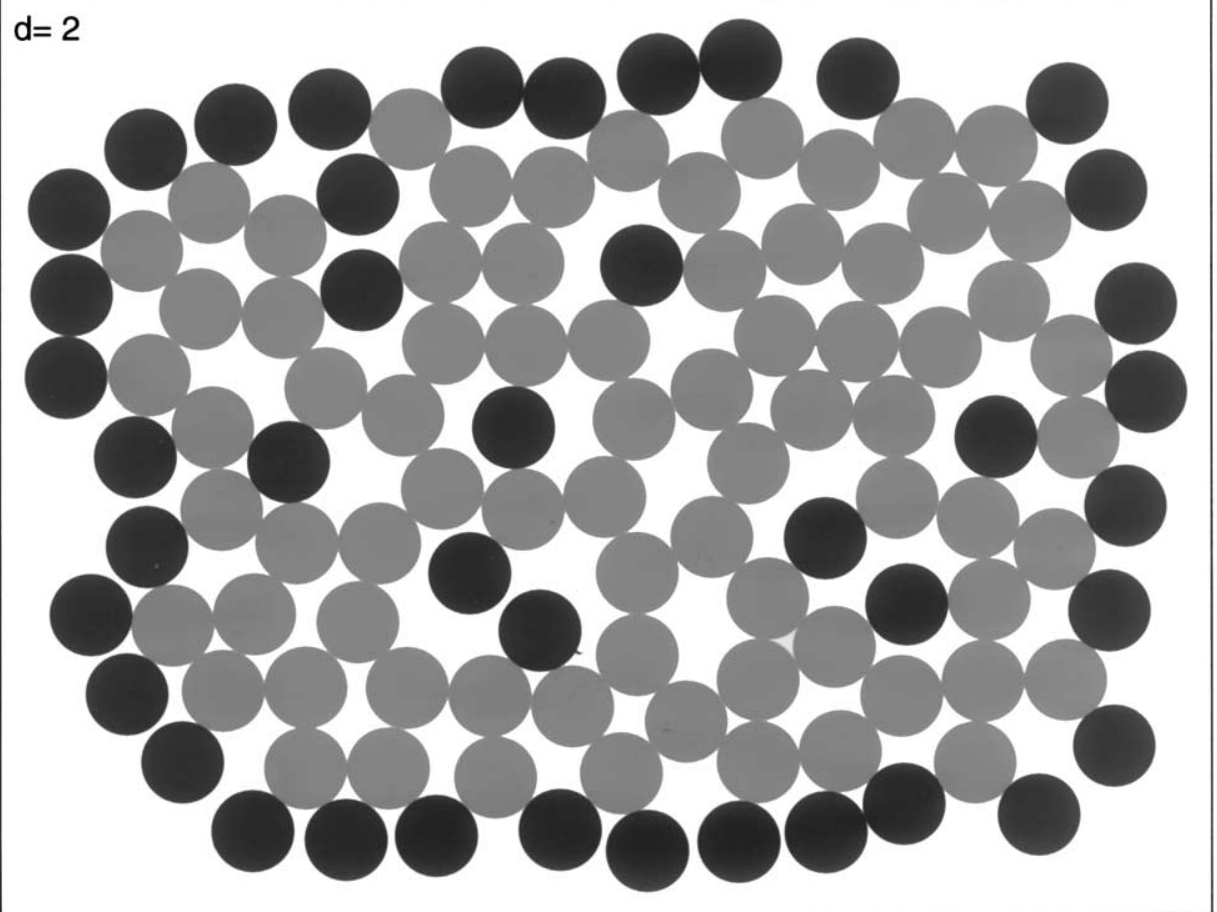

Fig. 2. (A) Contacts are placed on a $d$-1 dimensional sphere surface so for $d=1$ no distinction exists between 'caging' and 'kissing'; the caging density corresponds to one row of spheres that are all caged by the maximum of two neighbors except for the dark, uncaged boundary spheres. (B) At higher dimensions caging co-ordination numbers become distributed and also uncaged dark spheres may be present away from the boundary. A schematic is shown for an amorphous solid of 2- $d$ discs at the caging density, i.e. the average contact number equals the caging number $\left\langle N_{2}\right\rangle=3.35$. Since a 2- $d$ cage requires at least three contacts, and four contacts virtually always cage a disc, the disc cage in a plane contains either 3 or 4 contacts. The caged discs form a 2- $d$ queue (see text); collective vibrations would nevertheless crystallize the solid to the densest 2- $d$ packing.

This result can be seen as a geometrical equation of state for an amorphous solid of caged spheres: the caging number only depends on the Euclidean dimension $d$ which therefore completely fixes the caging density. For the case of uncorrelated contacts we find

$\Phi_{d}=\frac{2 d+1}{2^{d}}$

This is an exact result for the caging density for interpenetrating spheres, where each overlap blocks a translation direction of a test sphere. Due to such overlaps the density $\Phi_{d}$ may be larger than unity. For non-overlapping hard spheres we substitute the nummerical result from Eq. (2):

$\Phi_{d}=\frac{0.046 d^{2}+1.22+0.73}{2^{d}}$

which equals unity for the one-dimensional spheres in Fig. 2(A).

\section{Discussion}

The strong decrease of caging densities from Eq. (9) with Euclidean dimension, paralleling the trend in densities of lattice packings (Fig. 3), results from the competition between the nearly linear increase 


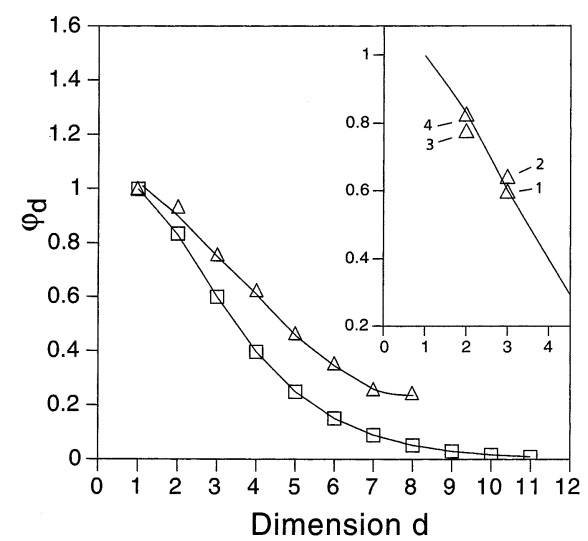

Fig. 3. Comparison between mean-field hard-sphere caging densities ( $\square$ ) from Eq. (9) for amorphous sphere solids and the densest possible sphere lattice packings[21] $(\Delta)$ as a function of dimension. Drawn lines are eye-guides. The insert compares caging densities (drawn line) to densities of (1) Scott's random loose sphere packing [13], (2) a simulated random close sphere packing [18], (3) a simulated random disc packing [15] and the average (4) from 12 studies on random disc packings [12].

of the caging number with dimension in Eq. (2) and the exponential growth of the excluded sphere volume. The latter produces the term $2^{-d}$ in Eq. (9), which always brings down the caging density at higher dimensions. Such a decrease also occurs for experimental amorphous solids in the form of random sphere packings; the random packing $(r p)$ density of static spheres is in 3-d substantially lower than in $2-d$. This previously unexplained dimensionality effect agrees actually quite well with Eq. (9), see insert of Fig. 3. Berryman's review [12] of $r p$-densities of discs on a twodimensional plane yields an average surface fraction of $\Phi_{2}=0.835( \pm 0.025)$, whereas Eq. (9) predicts a two-dimensional caging density of $\Phi_{2}=0.839$. Further, the three-dimensional caging volume fraction $\Phi_{3}=0.601$ equals the experimental value of $\Phi_{3}=0.60$ for the 'loose' $r p$-density first found by Scott [13].

It is somewhat unexpected that a local caging analysis would reproduce global packing densities. Moreover, in our analysis the only excluded volume effect is that for a pair of spheres in Eq. (4), which is a severe approximation. Therefore the agreement as shown in the insert of Fig. 3 should be viewed with some caution. There is, never- theless, no doubt that local caging effects are important in sphere packings; the question is how far they determine the final density irrespective of the packing preparation. Scott in his classical experiment [13] poured large numbers of uniform steel balls, which obviously 'settle' at an infinite rate on their diffusion time scale, into various containers and extrapolated densities to infinite container size. This pouring of spheres clearly is a complicated collective process that no more than the dynamics of glassy colloids can be modeled by a local geometrical caging analysis. However, the reproducible outcome of the process may well be a geometric state determined by minimal cage sizes, in the sense that the contact number of poured spheres jumps to the caging number, with boundary spheres caged due to container walls, keeping the spheres in a threedimensional queue (see below). The non-thermal spheres remain in this queue when left undisturbed and only densify (to random close packing) due to external vibrations [20].

If the majority of spheres in a packing or (static) glass are indeed locally caged, one could imagine that the contact number averaged over all spheres in a packing equals (or at least approximates) the average of a large number of individual caging experiments as depicted in Fig. 1(A). In that case the spheres in a packing would sample all possible outcomes of a repeated, single caging experiment. Contact numbers of sphere packings actually support this idea. For discs randomly packed in a plane an upperbound contact number of 3.416 has been reported [14] which is slightly above the two-dimensional caging number $\left\langle N_{2}\right\rangle=3.35$. Simulations of random disc packings of Hinrichsen et al. [15] produce an average contact number $\left\langle C_{2}\right\rangle=3.02$ and a density such that $\left\langle C_{2}\right\rangle / \varphi_{2}=3.9$ $( \pm 0.2)$, which agrees very well with the value of $2^{2}=4$ from Eq. (6). The three-dimensional caging number [16] is $\left\langle N_{3}\right\rangle=4.79$, which should be compared to the average contact number for the loose sphere packing found from simulations [22] to be $\left\langle C_{3}\right\rangle=5.28$, which increases [22] to 5.68 at the random close packing density of $64 \%$.

It should be noted that the 3- $d$ contact- and caging numbers are substantially below the 3-d random parking number [19] of about 8.7 (Fig. 
1(A)), which illustrates once more that an amorphous solid of caged spheres does not efficiently fill space. The solid does not maximize the number of parking places but instead - to stay in traffic jargon - employs minimal contact numbers to form a $d$-dimensional queue in which each caged sphere has to wait for one (and only one) particular contact to disappear and open its cage (Fig. 2). Whether such queue - when thermal motion is switched on - will form a long-lived state depends on the mobility of particles at the boundaries (Fig. 2), as in any traffic jam, and any frictional or cohesive forces between queuing colloids. Such effects, of course, cannot be derived solely from the geometry of hard-sphere cages composed only of central (point) forces between spheres.

Here we have explored the caging density for amorphous spheres as a function of Euclidean dimension. One can also change the particle shape at a given dimension, keeping the system amorphous both with respect to particle position as well as particle orientation. An interesting limiting case is a solid composed of randomly oriented thin rigid rods. The mean-field caging density for such a solid, based on Eq. (3) but now with the orientationally averaged excluded volume of a thin rod, is discussed elsewhere[23-25], including an exact solution for the caging number for a thin cylinder [24]. It turns out that this caging density for thin rods predicts the correct aspect-ratio dependence of experimental random rod packing densities [23] in the limit of high aspect ratio's. This agreement, which we also found in accurate computer simulations of random non-sphere packings [26], confirms that local caging effects are important for the calculation of random packing densities

\section{Conclusions}

It is possible to assign an approximate caging density to a static amorphous sphere solid, based on a precisely defined minimal cage size, which forms a contrast to the maximization represented by kissing and parking numbers. Caging densities and caging numbers at least qualitatively agree with known densities and contact numbers for random (loose) sphere packings in two and three dimensions. Also in view of the correct prediction for the aspect-ratio dependence of random thinrod packings it can be concluded that minimal cages are an important feature of random packings in general, and mutatis mutandis of systems such as sphere (or rod) glasses in as far as their static structure mimics such a random packing. The conversion from cage sizes to caging densities clearly needs to be improved beyond the meanfield approximation to further quantify the comparison to experimental sphere systems.

\section{Acknowledgements}

Theo Barenbrug, Gijsje Koenderink, Willem Kegel, Hans Melissen and Stephen Williams are acknowledged for enlightening discussions on geometry and sphere caging. Ingrid van Rooyen prepared the figures. This work is part of the research program of the Foundation for fundamental research on Matter (FOM) with financial support from the Netherlands Organization for Scientific Research (NWO).

\section{References}

[1] P.N. Pusey, W. Megen, Observation of a glass transition in suspensions of spherical colloidal particles, Phys. Rev. Lett. 59 (1987) 2083-2086.

[2] W.K. Kegel, A. van Blaaderen, Direct observation of dynamical heterogeneities in colloidal hard-sphere suspensions, Science 287 (2000) 290-293.

[3] J.P. Hansen, D. Levesque, J. Zinn-Justin (Eds.), Liquids, Freezing and the Glass Transition, North-Holland, Amsterdam, 1991.

[4] M Tokuyama, I. Oppenheim (Eds.), Air Conference Proceedings 469. Slow Dynamics in Complex Systems, American Institute of Physics, Woodbury, New York, 1999.

[5] B. Frick, R. Zorn, H. Büttner, Proceedings interworkshop on dynamics in confinement, J. Phys. IV 10 (2000) P37.

[6] A.P. Philipse, C. Pathmamanoharan, Liquid permeation (and sedimentation) of dense colloidal hard-sphere packings, J. Colloid Int. Sci. 159 (1993) 96-107.

[7] T.G. Mason, D.A. Weitz, Linear viscoelasticity of colloidal hard sphere suspensions near the glass transition, Phys. Rev. Lett. 75 (1995) 2770-2773. 
[8] R.S. Williams, I.K. Snook, W. van Megen, Molecular dynamics study of the stability of the hard sphere glass, Phys. Rev. E 02150664 (2001) 1-7.

[9] W. Schaertl, H. Sillescu, Brownian dynamics of polydisperse colloidal hard spheres: equilibrium structures and random close packing, J. Stat. Phys. 77 (1994) 1007-1025.

[10] H. Sillescu, Heterogeneity at the glass transition: a review, J. Non-crystalline Solids 243 (1999) 81-108.

[11] T. Aste, D. Weaire, The Pursuit of Perfect Packing, IOP Publishing, Bristol, 2000.

[12] J.G. Berryman, Definition of dense random packing, in: M. Shahinpoor (Ed.), Advances in the Mechanics and Flow of Granular Materials, vol. I, Gulf Publ. Company, Houston, TX, 1983, pp. 1-18.

[13] G. Scott, Packing of equal spheres, Nature 188 (1960) 908-909.

[14] W. Uhler, R. Schilling, A local description of stable 2D random packing, J. Phys. C: Solid State Phys. 18 (1985) L979-L983.

[15] E.L. Hinrichsen, J. Feder, T. Jøssang, Random packing of discs in two dimensions, Phys. Rev. A 41 (1990) 41994209.

[16] F. Peters, M. Kollmann, Th.M.A.O. Barenbrug, A.P. Philipse, Caging of a $d$-dimensional sphere and its relevance for the random dense sphere packing, Phys. Rev. E 02140463 (2001) 1-7.

[17] G. Mason, Radial distribution functions from small packings of spheres, Nature 217 (1968) 733-735.
[18] B. Lubachevski, F. Stillinger, E. Pinson, Disks vs. spheres: contrasting properties of random packings, J. Stat. Phys. 64 (1991) 501-524.

[19] M. Mansfield, L. Rakesh, D. Tomalia, The random packing of spheres on spheres, J. Chem. Phys. 105 (2) (1996) 3245-3249.

[20] G.D. Scott, D.M. Kilgour, The density of random close packing of spheres, Br. J. Appl. Phys. (J. Phys. D.) 2 (1969) $863-866$.

[21] J.H. Conway, N.J.A. Sloane, Sphere Packings, Lattices and Groups. Grundlehren der mathematischen Wissenschaften, Springer Verlag, New York, 1998, p. 290.

[22] D. He, N.N. Ekere, L. Cai, Computer simulation of random packing of unequal particles, Phys. Rev. E 60 (6) (1999) 7098-7104.

[23] A.P. Philipse, The random contact equation and its implications for colloidal rods in packings, supensions, and anisotropic powders, Langmuir 12 (1996) 1127-1133; Corrigendum Langmuir 12 (1996) 5971.

[24] A.P. Philipse, A. Verberkmoes, Statistical geometry of caging effects in random thin-rod structures, Phys. A 235 (1997) 186.

[25] A.P. Philipse, S.G.J.M. Kluijtmans, Sphere caging by a random fibre network, Phys. A 274 (1999) 516.

[26] S. Williams, A.P. Philipse, The random packing of nonspherical particles, (2002) submitted for publication. 